\title{
NOTICIAS
}

\section{Certificación y recertificación de médicos especialistas en salud pública}

El Consejo Nacional de Salud Pública, A.C. (CNSP), inició sus funciones el 6 de octubre de 2000. Obtuvo antes, el mes de julio, la Carta de Idoneidad por parte del Comité Normativo Nacional de Consejos de Especialidades Médicas. Lo que significa la magna resposabilidad que se ha impuesto el propio CNSP en sus estatutos para certificar y recertificar a los médicos especialistas en salud pública que se sometan al proceso de evaluación si así lo desean. La Mesa Directiva de este Consejo tomó posesión en la Sala Miguel Bustamante de la Secretaría de Salud, donde se expresó lo siguiente, por parte de los nuevos directivos:

- el objetivo único del CNSP para certificación y recertificación de los médicos especialistas en salud pública implica: a) el aseguramiento de la calidad en la formación de los mismos; $b$ ) la identificación del médico especialista en salud pública que responda a las necesidades de salud de la población, considerando las características del mercado de trabajo en el país y de acuerdo con los más recientes avances científicos y tecnológi- cos; c) disponer de la información sobre la calidad académica y administrativa para la acreditación de los programas de posgrado en el ámbito de la salud pública, y d) el seguimiento del proceso de acreditación voluntaria que hacen los programas de formación de recursos humanos;

- en la actualidad, la práctica de la salud pública no sólo se considera como el conjunto de prácticas socialmente organizadas, planeadas y ejecutadas por profesionales y técnicos para la promoción de la salud y la prevención de las enfermedades, sino que también se ha consolidado como proceso de investigación y de desarrolo de recursos humanos. El médico especialista en salud pública es capaz de generar y diseminar conocimientos científicos y es el más indicado para administrarlos eficazmente en la docencia y para aplicarlos eficientemente en el desarrollo de infraestructuras y programas de salud colectiva;

- el CNSP está suficientemente informado para elaborar y actualizar, por conducto de sus consejeros académicos, el sistema e instrumentos de evaluación de conocimientos, habilidades, actitudes y experiencia en el campo de la salud pública a fin de asegurar la calidad profesional de los médicos especialistas en esta área que soliciten su certificación y recertificación;

- los consejeros académicos, además de asesorar a la Mesa Directiva en general y al secretario académico en particular, participan activamente en la elaboración de los programas de evaluación del médico especialista en salud pública, integran el jurado examinador de los médicos que sean sometidos al proceso de evaluación y garantizan con su voto la idoneidad de la certificación de los médicos aspirantes;

- para definir los criterios de certificación de esta especialidad médica, el CNSP tiene en cuenta los criterios, instrumentos y reglamentos para la acreditación de los programas de posgrado en esta área del conocimiento, de las escuelas y facultades pertenecientes a la Asociación Mexicana de Educación en Salud Pública (AMESP), en particular los relacionados con la contribución para la definición de políticas y programas sobre la formación de recursos humanos idóneos y la realización de actividades de investigación en los sectores 
de la salud y educación en el país;

- con la orientación del Instituto Nacional de Salud Pública (INSP) se ha revisado la información pertinente sobre las normas y patrones de calidad existentes en relación con los planes de estudio, personal académico, ingreso, acreditación y obtención de grado académico de los estudiantes, sistemas de indicadores de evaluación y otras características de la educación de posgrado. También se han consultado los documentos relacionados con las normas vigentes para los posgrados nacionales de la Secretaría de Educación Pública, los de la Comisión Nacional de Evaluación de la Educación Superior y el de Acreditación de programas académicos de posgrado en salud pública (AMESP-OPS/OMS*,2000);

- asimismo, revisamos el catálogo de programas de posgrado en salud pública (4a. versión, 2000), compilado por la AMESP y el INSP, con la cooperación técnica de la OPS/OMS, en el cual se ordenan los programas e infraestructura dedicados a la enseñanza en salud pública y se analiza la capacidad disponible de acuerdo con las debilidades y fortalezas de cada institución y las características propias de los cursos y grados que se otorgan y como responden a la problemática y prioridades locales y regionales de salud colectiva, en el marco del Sistema Nacional de Enseñanza en Salud Pública;

- los programas de doctorado en salud pública son ofrecidos únicamente por el INSP y la Uni-

\footnotetext{
* Organización Panamericana de la Salud/
} Organización Mundial de la Salud. versidad de Guadalajara. En ambas instituciones, la duración es de 24 a 70 meses. En los estudios de maestría, el total de horas osciló entre 960 y 2 756. En ambos posgrados la titulación se condiciona a la presentación de tesis. Las especialidades se imparten únicamente en el INSP y en tres universidades: Universidad Juárez del Estado de Durango, la Universidad Veracruzana y la Universidad Autónoma de Chihuahua. Las instituciones condicionan la titulación a formas diversas como la tesina, examen y práctica de campo o tesis colectiva;

- el CNSP tiene acceso permanente a los programas académicos actualizados, los cuales son revisados para afinar los criterios de certificación y recertificación de médicos especialistas en salud pública, y

- el CNSP guarda estrecha relación con la Sociedad Mexicana de Salud Pública, la cual está integrada por filiales estatales, cuyos miembros son, en gran proporción, médicos sanitaristas con educación de posgrado y sólida experiencia en programas y estructuras de salud, quienes han manifestado su deseo de ser evaluados por el CNSP para su certificación.

Francisco Javier López Antuñano, Investigador titula Instituto Nacional de Salud Pública, México.

\section{Actividades del Instituto de Diagnóstico y Referencia Epidemiológicos de México}

El Instituto de Diagnóstico y Referencia Epidemiológicos (InDRE) se originó como Instituto de Salubri- dad y Enfermedades Tropicales (ISET), en 1939. Ha tenido a lo largo de su historia dos orientaciones diferentes: desde su creación hasta 1985 fungió como hospital para enfermedades tropicales sujetas a proyectos de investigación clínica y contó con médicos de gran reconocimiento nacional e internacional. Esta actividad, a lo largo de los años, fue decayendo y la retomaron paulatinamente otros institutos que se fueron creando en ese tiempo. En 1985, para apoyar los programas de vigilancia epidemiológica, se dio prioridad al desarrollo y ejecución de técnicas eficientes para el diagnóstico y la referencia, al fortalecimiento de redes nacionales de diagnóstico específico y a la generación de la Red Nacional de Laboratorios de Salud Pública (RNLSP). Bajo estos nuevos criterios, en 1989, el Instituto cambió su nombre por Instituto Nacional de Diagnóstico y Referencia Epidemiológicos Doctor Manuel Martínez Báez e inició la incorporación de las redes de diagnóstico de tuberculosis, paludismo y cáncer cervicouterino, que funcionaban de manera independiente, y se implementó la red de virus de inmunodeficiencia humana (VIH) para bancos de sangre. A raíz de la última pandemia de cólera que llegó a México en junio de 1991, el InDRE tuvo una actividad muy relevante y organizó la habilitación de laboratorios en todo el país para la identificación del Vibrio cholerae.

Actualmente, el InDRE está organizado en 16 departamentos y cuenta con 69 laboratorios. Los objetivos institucionales son: a) realizar diagnóstico de laboratorio mediante tecnología de uso común o de vanguardia; b) realizar la coordinación técnica de la RNLSP; c) establecer estándares de calidad y seguridad en los laboratorios; d) evaluar, generar y adaptar tecnología apropiada de diagnóstico; e) promover el desarrollo de recursos humanos y la capaci- 
tación; f) desarrollar investigaciones básicas y aplicadas, y e) apoyar el proceso de descentralización y el programa nacional de supervisión integral. El gran crecimiento del InDRE ha conllevado un aumento de $40 \%$ en las muestras que se han recibido a partir de 1995 que actualmente suman más de 220000 por año. Esto implicó la creación de una red informática, denominada INFO$\mathrm{LAB}$, que tiene capacidad de ser actualizada según necesidades, lo que ha permitido incorporar la información clínica de las muestras y emitir con oportunidad los resultados de laboratorio. De las 226087 pruebas realizadas en 1998, 54006 correspondieron al control de calidad de gota gruesa de paludismo; 53 101, a la confirmación de Aedes aegypti, y 15921 fueron laminillas de material clínico teñidas por la técnica de Papanicolaou, para su selección e inclusión en el control de calidad externo a la red correspondiente. Por otro lado, 7232 pruebas en el área de cólera y diarreas fueron de referencia para la caracterización bioquímica y molecular de las cepas de Salmonella spp, Shigella spp y V. cholerae aisladas en los Laboratorios Estatales de Salud Pública (LESP), 4874 muestras fueron para realizar cultivos y evaluación de sensibilidad a drogas de Mycobacterium tuberculosis y 2289 sueros para confirmación de la infección por el VIH por inmunoelectrotransferencia, la mayoría de los cuales fueron enviados por los LESP. Los estudios para la identificación de genes de agentes infecciosos y sus productos en 3820 muestras y los 698 análisis inmunogenéticos, por ser muy especializados únicamente se realizan en el InDRE, así como las 10057 consultas de la sección de dermatología. Por lo tanto, más de $60 \%$ de todas las pruebas efectuadas en el Instituto fueron para control de calidad o referencia, lo cual es congruente con el papel institucional como centro rector de la RNLSP en muestras de seres humanos, de reservorios y de vectores. Los diagnósticos que se siguen realizando en el InDRE están dirigidos principalmente a enfermedades emergentes, remergentes, de distribución limitada, o que por acuerdos internacionales únicamente se pueden realizar en el Instituto, así como aquellos que requieren de técnicas o equipos complejos, costosos o que aún no están disponibles en todos los LESP. El personal técnico del Instituto, bajo las normas del aseguramiento de la calidad, utiliza cotidianamente las bitácoras de trabajo y los manuales de procedimientos y de organización y maneja adecuadamente los residuos peligrosos biológicos infecciosos (RPBI) y los químicos.

Las pruebas que emplea la RNL$\mathrm{SP}$ tienen diversos grados de complejidad, pueden ser mínimas, como las que se realizan en los laboratorios locales, generales, que requieren de equipos más costosos, o técnicas más complejas, las cuales se realizan en los LESP y, especiales, que se llevan a cabo sólo en el InDRE. Las primeras también se pueden utilizar en los niveles estatal y nacional y algunas especiales en el estatal. Para llevar a cabo sus funciones de manera óptima, es importante que los LESP estén ubicados en edificios apropiados y que tengan equipos, reactivos y materiales suficientes. Sin embargo, existe una gran heterogeneidad en la infraestructura de que disponen; aproximadamente una tercera parte tiene instalaciones y equipamiento óptimos, pero otro tanto no cuenta con un local específico y se apoya en unidades especializadas dispersas. La importancia dada a la confirmación diagnóstica en apoyo a la vigilancia epidemiológica se hace evidente por los ocho LESP, inaugurados entre 1994 y 1998, y los cuatro que están actualmente en construcción. Los laboratorios locales, por su carácter individual, realizan pruebas que requieren de equipo mínimo, por ejemplo, microscopio óptico, que se pueden usar para diagnóstico de tuberculosis, paludismo, cáncer cervicouterino e infecciones presentes en exudados urogenitales, así como pruebas que no requieren de equipo de lavado o de lectura (pruebas rápidas) como son las de aglutinación, ELISA en punto o inmunocromatografía para la infección por VIH, dengue, brucelosis, etcétera. Otras unidades utilizan equipo de uso sencillo para identificación de bacterias o de rotavirus causantes de diarreas. Las excepciones son los laboratorios que realizan ELISA para VIH, dengue y enfermedad febril exantemática y los de diagnóstico de rabia, ya que requieren de un lector de placas o un microscopio de epifluorescencia, respectivamente, que son costosos y de manejo especializado. Debido a la aparición continua de nuevos estuches diagnósticos en el mercado, el InDRE, a solicitud de la industria nacional y del extranjero, los evalúa para demostrar su calidad y eficiencia.

Para constatar que las actividades descentralizadas se llevan a cabo con eficiencia y de acuerdo con las normas establecidas, la Subsecretaría de Prevención y Control de Enfermedades organizó un programa nacional de supervisión integral. El InDRE participa con visitas a los LESP y a laboratorios locales, y genera recomendaciones que permiten mejorar su funcionamiento como servicios descentralizados. Otro aspecto relacionado es el apoyo que brinda el Instituto cuando acontecen desastres naturales y aparecen urgencias epidemiológicas, en las cuales interviene fundamentalmente en el diagnóstico de infecciones por enterobacterias, el aislamiento y caracterización de agentes de fiebres 
hemorrágicas (dengue y leptospirosis) y en la vigilancia entomológica de vectores, además, se organizan laboratorios de campo en las regiones afectadas o se refuerzan con reactivos a los existentes. Ejemplos recientes de lo anterior fueron las actividades realizadas en relación con los huracanes Paulina, en las costas de los estados de Guerrero y Oaxaca, en 1997, y Mitch en el sudeste del país, en 1998; en este último también se dio apoyo a países de Centroamérica.

También se ha impulsado el adiestramiento del personal del InDRE en instituciones de prestigio, tanto nacionales como en el extranjero, y en cursos colectivos monográficos y especializados. Estos últimos son apoyados por la Facultad de Medicina de la Universidad Nacional Autónoma de México, por ser el InDRE uno de sus centros de educación médica continua, a los que asisten, además del propio personal, miembros de la RNLSP y profesionales externos. Asimismo, se organizan cursos y talleres internos sobre conocimientos básicos, discusión de protocolos y datos de investigación, elaboración de manuscritos y seminarios con presentaciones de científicos sobresalientes nacionales y del extranjero, y personal del Instituto. Se ha incrementado la participación del personal del InDRE y de los LESP en congresos, reuniones, simposios y talleres, así como las capacitaciones en servicio del personal de la RNLSP según la solicitud de cada entidad federativa.
En lo relacionado con investigación, se realizan protocolos en áreas muy diversas que se pueden resumir en: desarrollo de tecnologías diagnósticas basadas principalmente en métodos inmunológicos o moleculares; estudios epidemiológicos de brotes y de muestreos locales y nacionales; caracterización molecular de agentes infecciosos prevalentes en México; respuesta inmunológica y estudios de susceptibilidad en enfermedades infecciosas y no infecciosas, y estudios poblacionales de artrópodos e identificación de vectores de importancia médica. De los proyectos de investigación en progreso, $60 \%$ son llevados a cabo en colaboración con instituciones nacionales del sector salud o de educación superior y $23 \%$ con instituciones de otros países. Del presupuesto que se ejerce para investigación, $18 \%$ proviene de financiamiento externo, la mitad de agencias financiadoras mexicanas y la otra parte del extranjero, siendo en este caso Estados Unidos de América el país que más aporta. También se llevan a cabo proyectos de cooperación técnica internacional con apoyo de la Organización Panamericana de la Salud, que incluyen transferencia, desarrollo o validación de tecnología.

La difusión de la información de interés científico se presenta en reuniones científicas o se publica en forma de comunicaciones originales, en revistas de distribución nacional e internacional. En su mayoría, estas publicaciones provienen del análisis de los datos de los protocolos de investigación aprobados, pero también se ha generado información a partir del trabajo de diagnóstico o referencia de rutina, que, además de apoyar la vigilancia epidemiológica y la toma de decisiones, ha dado a conocer diversos aspectos de las enfermedades con diagnóstico de laboratorio en foros nacionales o internacionales. El InDRE también tiene como prioridad la elaboración y actualización de manuales de procedimientos de laboratorio; recientemente se publicaron los de diagnóstico de enfermedad febril exantemática, parálisis flácida aguda, micosis, tuberculosis, zoonosis, cáncer cervicouterino, así como para procedimientos de histocompatibilidad, genética molecular y anticuerpos monoclonales.

\section{Agradecimientos}

Los autores agradecen el apoyo de todo el personal del InDRE y de la RNLSP por el cumplimiento de su trabajo con entusiasmo y calidad. Al Q.B.P. Fernando González y al señor Alberto Carreto por su valiosa colaboración.

\footnotetext{
Dra. Ana Flisser Steinbruch, Dr. Alejandro Escobar Gutiérrez, Dra. Dolores Correa Beltrán, Instituto de Diagnóstico y Referencia Epidemiológicos, Secretaría de Salud, México.
} 